\title{
Aplicación de la metodología enfermera a través del aprendizaje basado en problemas
}

\author{
MACARENA ROMERO-MARTÍN \\ Centro Universitario de Enfermería Cruz Roja \\ Universidad de Sevilla \\ mromero@cruzroja.es \\ ORCID: https://orcid.org/0000-0003-3022-3339 \\ D.O.I.: http://dx.doi.org/10.12795/JDU.2018.i01.99 \\ Pp.: $1756-1772$
}

\section{Resumen}

Se desarrolla un Ciclo de Mejora Docente se desarrolla en la asignatura Historia, Teorías y Método de la Enfermería II, en la titulación de Grado de Enfermería, para trabajar la aplicación práctica de las etapas de diagnóstico y planificación del Proceso Enfermero. Se centra en cómo llevar este proceso a la práctica clínica, e incluye contenidos teóricos básicos que permiten articular el proceso y competencias actitudinales. Se empleó la metodología docente de aprendizaje basado en problemas. El problema de partida fue una situación clínica a la que el alumnado debe dar respuesta como profesional de enfermería. El sistema de aprendizaje basado en casos clínicos ha mostrado ser efectivo especialmente para los contenidos actitudinales. En relación con la satisfacción del alumnado, el mayor de grado de acuerdo se obtuvo al afirmar que el ABP potencia el trabajo en equipo, facilita el pensamiento crítico y logra una mejor comprensión de la materia. 
Palabras clave: Historia, Teorías y Método de la Enfermería II, Grado en Enfermería, docencia universitaria, experimentación docente universitaria, aprendizaje basado en problemas.

\section{Diseño previo del ciclo de mejora docente}

\section{Contextualización de la intervención}

El presente Ciclo de Mejora Docente (CMD) se desarrolla en la asignatura Historia, Teorías y Método de la Enfermería II, que se imparte en el primer cuatrimestre de segundo curso de Grado de Enfermería, en el Centro Universitario de Enfermería Cruz Roja, adscrito a la Universidad de Sevilla. La a signatura cuenta con 6 créditos ECTS, cuya docencia presencial se distribuye en 50 horas de clases teóricas y 10 horas de clases prácticas. En las clases teóricas participan todos los/as alumnos/as matriculados, conformando un grupo de 70 personas, y para las clases prácticas, se forman 5 grupos de 14 participantes cada uno. La asignatura se centra en el método de organización de la práctica enfermera asistencial, el Proceso Enfermero (PE) y su aplicación en casos clínicos. El PE es un proceso secuencial que facilita una atención integral a las personas y familias en respuesta a sus necesidades de salud. Consta de cinco pasos interrelacionados: valoración, diagnóstico, planificación, ejecución y evaluación. Se pretende que el alumnado sea capaz de identificar y desempeñar cada una de las etapas ante supuestos casos clínicos extraídos de la realidad asistencial. El CMD propuesto tiene una duración de 12 horas y se desarrolla en 5 sesiones teóricas de grupo completo entre las fechas 31 de octubre y 16 de noviembre.

Jornadas de Formación e Innovación Docente del Profesorado | № 1 (2018) Esta obra se distribuye con la licencia Creative Commons 


\section{Mapa de contenidos}

El presente CDM se va a emplear para trabajar la aplicación práctica de las etapas de diagnóstico y planificación del PE. Se centra en cómo llevar el proceso a la práctica clínica, aunque incluye los contenidos teóricos básicos que permiten articular el proceso (definiciones de conceptos nucleares y las relaciones entre los conceptos) y las competencias actitudinales transversales. Los contenidos conceptuales están resaltados en azul, los procedimentales en verde y los actitudinales en amarillo. La figura 1 reúne los contenidos que pretenden aprender:

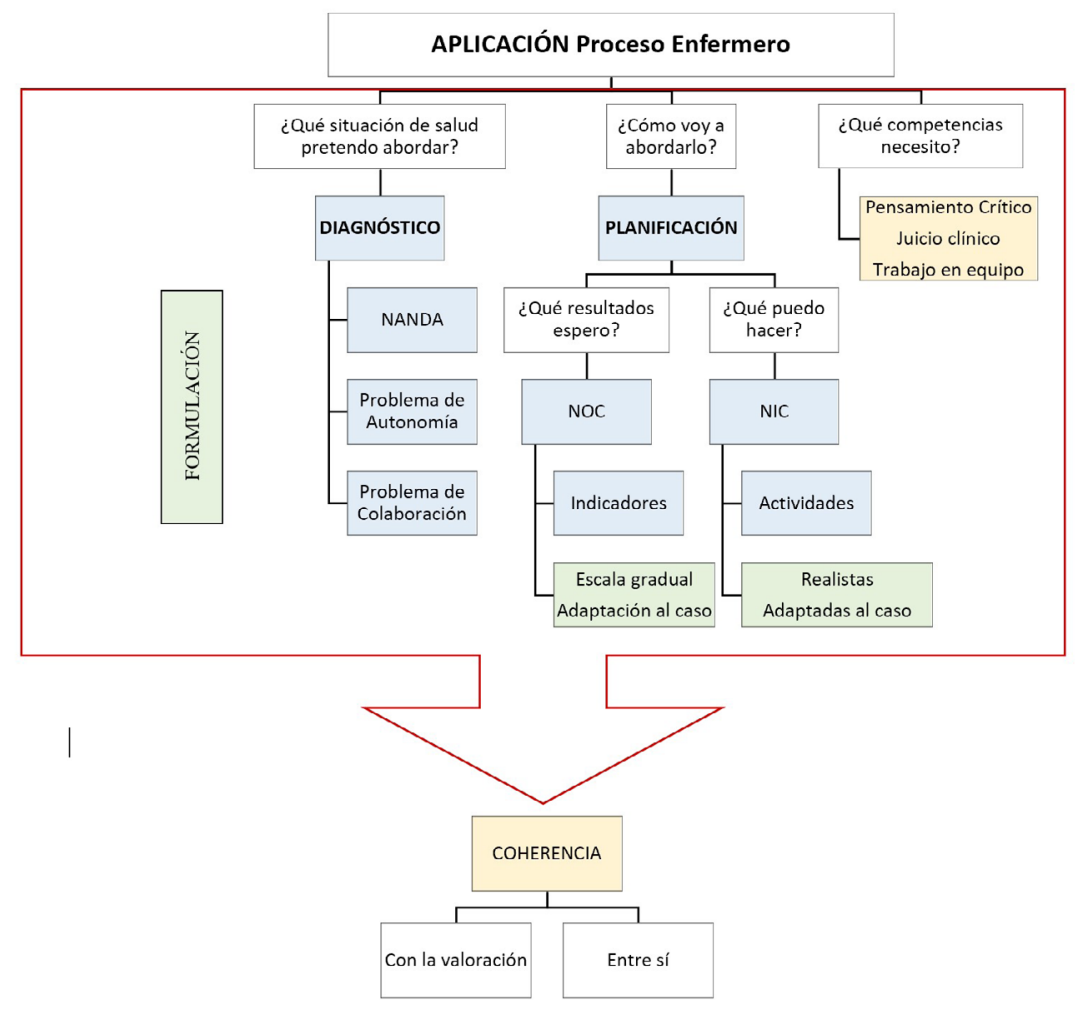

Figura 1. Mapa de contenidos.

Jornadas de Formación e Innovación Docente del Profesorado | № 1 (2018)

(i) Esta obra se distribuye con la licencia Creative Commons Reconocimiento-NoComercial-SinObraDerivada Internacional (CC BY-NC-ND 4.0.) 


\section{Modelo docente de partida}

Hasta ahora, la aplicación práctica del PE seguía un modelo unidireccional por imitación. En las clases teóricas de grupo completo, la profesora explica los conceptos, los pasos del PE y cómo se elabora. Se muestran ejemplos de casos resueltos y se resuelve un caso en común, dirigido por la profesora resolviendo las dudas. A continuación, los estudiantes resuelven supuestos casos clínicos propuestos por la profesora de forma autónoma, respondiendo a unos cuestionarios a través del campus virtual. Las preguntas del cuestionario se corrigen en común, aclarando los fallos. El protagonismo recae en la docente, que ejerce el papel de transmisora. El alumnado tiene un papel activo, pero con poca iniciativa. El conocimiento es concebido como algo externo que debe interiorizarse siguiendo unas pautas establecidas por el docente, imitando su modo de proceder.

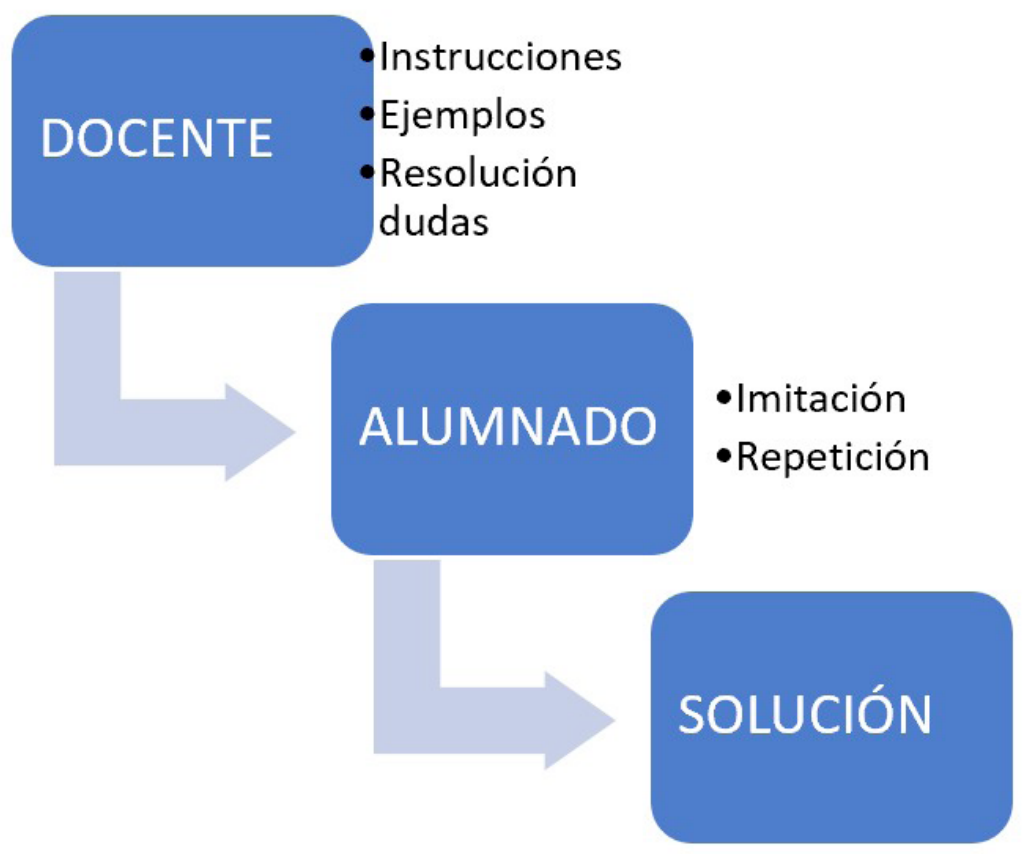

Figura 2. Modelo docente de partida.

Jornadas de Formación e Innovación Docente del Profesorado I № 1 (2018) Esta obra se distribuye con la licencia Creative Commons 


\section{Modelo metodológico ideal}

El propósito de este CMD es seguir un modelo basado en la reelaboración de ideas de los estudiantes. Un problema o incógnita que despierta el interés del alumnado sirve de punto de para elaborar una respuesta. Esta respuesta se comparte y compara con las de los demás o con otras fuentes de información, identifica sus errores o carencia y las complementa con sus aportes. De este modo, tanto estudiante como docente tienen un papel activo y el conocimiento se construye desde el interior. El aprendizaje es un proceso de elaboración de respuestas.

Para este fin, el CMD se empleará la metodología docente de aprendizaje basado en problemas con elementos de gamificación. El problema de partida será una situación clínica a la que el alumnado debe dar respuesta como profesional de enfermería aplicando el PE.

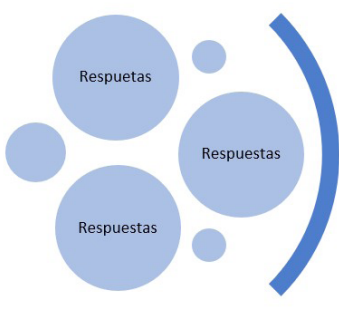

Problema (caso clínico)

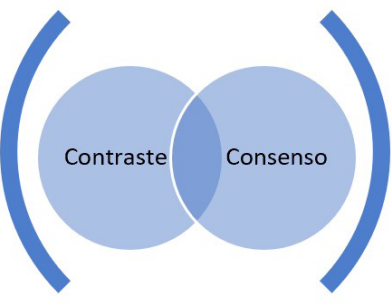

Trabajo en equipo

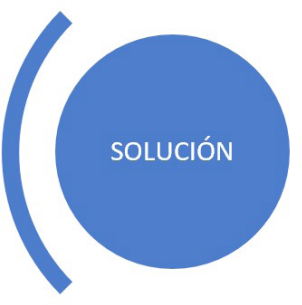

Docente $=$ orientador

Figura 3. Modelo metodológico ideal.

\section{Secuencia de actividades}

El presente CMD tiene un enfoque instrumental, de aplicación práctica de un procedimiento. Sin embargo, y a pesar de que se pretende huir de un modelo de transmisión de teoría seguido de la consecuente aplicación práctica, es preciso explicar inicialmente unos conceptos básicos. Tanto el diagnóstico enfermero como las taxonomías son conceptos totalmente ajenos al alumnado, no han sido trabajados previamente en ninguna otra asignatura, ni pertenecen a la cotidianeidad. Se propone iniciar 
el CMD con una sesión introductoria, aunque se debe aclarar que en esta sesión se trabajarán las definiciones y herramientas que pueden utilizar, y no cómo emplearlas, ni cómo se relacionan los conceptos entre sí. Esta parte instrumental es la que se pretende trabajar con aprendizaje basado en problemas.

Por lo tanto, se comenzará con una sesión teórica de 1 hora de duración en la que se informará al alumnado sobre el desarrollo del CMD y su participación en él. Se explicarán los conceptos básicos (diagnóstico enfermero, NANDA, diagnóstico de autonomía, problema de colaboración, NOC, indicadores, NIC y actividades) con una metodología de clase expositiva con apoyo audiovisual. Se anima ala participación del alumnado resolviendo las dudas.

Las seis sesiones siguientes se centran en la resolución de un caso clínico, que será el problema que desencadene el aprendizaje. El alumnado dispondrá del material de consulta teórico con anterioridad a la sesión y durante la misma. Se recomienda que el alumnado haya leído previamente este material. Los casos clínicos son diseñados con un grado de dificultad ascendente, para adaptarse al progreso del alumnado. Los tres primeros casos se centran exclusivamente en la etapa diagnóstico y los tres últimos abarcan además la etapa planificación. Cada sesión tendrá una duración de 2 horas y sigue una estructura similar:

1. Reagrupación de los asistentes en equipos de tres a cuatro personas. Los equipos se forman según afinidades personales, para facilitar la comunicación y dinámica entre ellos. Se formarán al inicio de la sesión y no se establecerán previamente porque la asistencia a los grupos completos no es obligatoria, por lo tanto, los equipos podrían no estar completos el día en que se celebra la sesión. Cada equipo elegirá un nombre que les identifique para la segunda parte de la sesión.

Jornadas de Formación e Innovación Docente del Profesorado I № 1 (2018) Esta obra se distribuye con la licencia Creative Commons 
2. Reparto de la documentación: cada equipo dispondrá de copias del caso clínico y fuentes de información donde documentarse sobre cómo resolverlo.

3. Pregunta intrigante y presentación del caso: se expone el caso clínico y se resuelven las dudas relacionadas con el vocabulario técnico (la asignatura se desarrolla en el primer cuatrimestre del segundo curso, el alunado aún no ha hecho prácticas clínicas y no está aún familiarizado con la terminología clínica).

4. Resolución del caso en equipo: cada grupo elabora una respuesta al problema planteado apoyándose en la documentación facilitada.

5. Puesta en común y corrección del caso: a través de un juego-concurso. Los equipos compiten con cuestionario on-line en tiempo real desde la plataforma Kahoot en la modalidad Quiz. Las preguntas se responden por equipo y el equipo que obtenga mayor número de respuestas acertadas ganará el concurso. Las preguntas se autocorrigen de forma inmediata, así que al final de cada pregunta se solicita a uno de los grupos que hayan acertado que argumente su respuesta y se aclaran así las dudas de los grupos que no hayan acertado. El premio consiste en la adjudicación automática de 0,1 puntos en la calificación final de la asignatura.

La siguiente tabla describe el perfil de los casos, con los que se trabajan todos los contenidos propuestos: 
Tabla 1. Casos clínicos de la fase diagnóstico: ¿Qué situación de salud pretendo abordar?

\begin{tabular}{|c|c|c|c|c|}
\hline & Dificultad & Contenidos & Resultados esperados & Pregunta inicial \\
\hline Caso 1 & Baja & $\begin{array}{l}\text { NANDA } \\
\text { Formulación } \\
\text { Pensamiento crítico } \\
\text { Juicio clínico } \\
\text { Trabajo en equipo } \\
\text { Coherencia con la } \\
\text { valoración }\end{array}$ & $\begin{array}{l}\text { Identificar la necesi- } \\
\text { dad alterada } \\
\text { Identificar dos diag- } \\
\text { nósticos NANDA reales } \\
\text { Formular } \\
\text { correctamente }\end{array}$ & $\begin{array}{l}\text { ERROR MÉDICO: } \\
\text { Ernesto Mate } \\
\text { tiene Diabetes } \\
\text { Mellitus I, pero } \\
\text { en realidad, ese } \\
\text { no es su diag- } \\
\text { nóstico. ¿Cuál } \\
\text { es su verdadero } \\
\text { diagnóstico? }\end{array}$ \\
\hline Caso 2 & Baja & $\begin{array}{l}\text { NANDA } \\
\text { Formulación } \\
\text { Pensamiento crítico } \\
\text { Juicio clínico } \\
\text { Trabajo en equipo } \\
\text { Coherencia con la } \\
\text { valoración }\end{array}$ & $\begin{array}{l}\text { Identificar la necesi- } \\
\text { dad alterada } \\
\text { Identificar un diagnós- } \\
\text { tico NANDA real y otro } \\
\text { de riesgo } \\
\text { Formular } \\
\text { correctamente }\end{array}$ & $\begin{array}{l}\text { ERROR MÉDICO: } \\
\text { Carmelo Co- } \\
\text { ton tiene Hiper- } \\
\text { tensión Arterial, } \\
\text { pero en rea- } \\
\text { lidad, ese no } \\
\text { es su diagnós- } \\
\text { tico. ¿Cuál es } \\
\text { su verdadero } \\
\text { diagnóstico? }\end{array}$ \\
\hline Caso 3 & Media & $\begin{array}{l}\text { Problema de } \\
\text { autonomía } \\
\text { Problema de } \\
\text { colaboración } \\
\text { Formulación } \\
\text { Pensamiento crítico } \\
\text { Juicio clínico } \\
\text { Trabajo en equipo } \\
\text { Coherencia con la } \\
\text { valoración }\end{array}$ & $\begin{array}{l}\text { Identificar la necesi- } \\
\text { dad alterada } \\
\text { Identificar un pro- } \\
\text { blema de autonomía y } \\
\text { otro de colaboración } \\
\text { Formular } \\
\text { correctamente }\end{array}$ & $\begin{array}{l}\text { ERROR MÉDICO: } \\
\text { Germán Tequilla } \\
\text { tiene ELA, pero } \\
\text { en realidad, ese } \\
\text { no es su diag- } \\
\text { nóstico. ¿Cuál } \\
\text { es su verdadero } \\
\text { diagnóstico? }\end{array}$ \\
\hline
\end{tabular}


Tabla 2. Casos clínicos de la fase planificación: ¿Cómo voy a abordarlo? ¿Qué resultados espero? ¿Qué puedo hacer?

\begin{tabular}{|c|c|c|c|c|}
\hline & $\begin{array}{c}\text { Dificul- } \\
\text { tad }\end{array}$ & Contenidos & Resultados esperados & Pregunta inicial \\
\hline $\begin{array}{l}\text { Caso } \\
4\end{array}$ & Media & $\begin{array}{l}\text { NANDA } \\
\text { NOC } \\
\text { Indicadores } \\
\text { NIC } \\
\text { Actividades } \\
\text { Pensamiento } \\
\text { crítico } \\
\text { Juicio clínico } \\
\text { Trabajo en equipo } \\
\text { Formulación } \\
\text { Coherencia con la } \\
\text { valoración } \\
\text { Coherencia en- } \\
\text { tre sí } \\
\text { Adaptación al } \\
\text { caso } \\
\text { Realismo } \\
\text { Graduación } \\
\text { correcta }\end{array}$ & $\begin{array}{l}\text { Identificar la necesidad } \\
\text { alterada } \\
\text { Identificar un diagnós- } \\
\text { tico NANDA real } \\
\text { Formular correctamente } \\
\text { Identificar un NOC } \\
\text { Tres indicadores } \\
\text { Una NIC } \\
\text { Tres actividades }\end{array}$ & $\begin{array}{l}\text { Aitor Tilla tiene obe- } \\
\text { sidad ¿qué pode- } \\
\text { mos hacer por él? }\end{array}$ \\
\hline $\begin{array}{l}\text { Caso } \\
5\end{array}$ & Alta & $\begin{array}{l}\text { NANDA } \\
\text { NOC } \\
\text { Indicadores } \\
\text { NIC } \\
\text { Actividades } \\
\text { Pensamiento } \\
\text { crítico } \\
\text { Juicio clínico } \\
\text { Trabajo en equipo } \\
\text { Formulación } \\
\text { Coherencia con la } \\
\text { valoración } \\
\text { Coherencia en- } \\
\text { tre sí } \\
\text { Adaptación al } \\
\text { caso } \\
\text { Realismo } \\
\text { Graduación } \\
\text { correcta }\end{array}$ & $\begin{array}{l}\text { Identificar la necesidad } \\
\text { alterada } \\
\text { Identificar un diagnós- } \\
\text { tico NANDA real } \\
\text { Formular correctamente } \\
\text { Identificar un NOC } \\
\text { Tres indicadores } \\
\text { Una NIC } \\
\text { Tres actividades }\end{array}$ & $\begin{array}{l}\text { Encarna Vales ha } \\
\text { optado por la lac- } \\
\text { tancia materna, } \\
\text { ¿qué podemos ha- } \\
\text { cer por ella? }\end{array}$ \\
\hline
\end{tabular}




\begin{tabular}{|c|c|c|c|c|}
\hline $\begin{array}{l}\text { Caso } \\
6\end{array}$ & Alta & $\begin{array}{l}\text { NANDA } \\
\text { NOC } \\
\text { Indicadores } \\
\text { NIC } \\
\text { Actividades } \\
\text { Pensamiento } \\
\text { crítico } \\
\text { Juicio clínico } \\
\text { Trabajo en equipo } \\
\text { Formulación } \\
\text { Coherencia con la } \\
\text { valoración } \\
\text { Coherencia en- } \\
\text { tre sí } \\
\text { Adaptación al } \\
\text { caso } \\
\text { Realismo } \\
\text { Graduación } \\
\text { correcta }\end{array}$ & $\begin{array}{l}\text { Identificar la necesidad } \\
\text { alterada } \\
\text { Identificar un diagnós- } \\
\text { tico NANDA real } \\
\text { Formular correctamente } \\
\text { Identificar un NOC } \\
\text { Tres indicadores } \\
\text { Una NIC } \\
\text { Tres actividades }\end{array}$ & $\begin{array}{l}\text { Maruja Ron tiene } \\
\text { pérdida de orina, } \\
\text { ¿qué podemos ha- } \\
\text { cer por ella? }\end{array}$ \\
\hline
\end{tabular}

\section{Seguimiento de la evolución de los conocimientos}

La evolución de adquisición de contenidos se determinará evaluando cada caso clínico realizado por el alumnado. Los casos pueden dividirse en dos bloques de tres casos cada uno, atendiendo a la etapa del PE en la que se centran: caso 1, 2 y 3 fase diagnóstico y caso 4, 5 y 6 fase planificación. De esta forma, el caso 1 hace las veces de cuestionario inicial del bloque diagnóstico, con la pregunta ¿Qué situación de salud pretendo abordar?. El caso 3 es el cuestionario final y el caso 2 puede considerarse una medida intermedia. En el bloque de planificación, el caso 4 hace las veces de cuestionario inicial, con la pregunta ¿Cómo voy a abordarlo? ¿Qué resultados espero? ¿Qué puedo hacer? El caso 6 es el cuestionario final y el caso 5 actúa como una medida intermedia. Para poder sintetizar, analizar y comparar los resultados se emplean las siguientes escaleras de aprendizaje para cada pregunta, y Así es posible medir, cuantificar y comparar la evolución de los contenidos del mapa. 
Pregunta: ¿Qué situación de salud pretendo abordar?

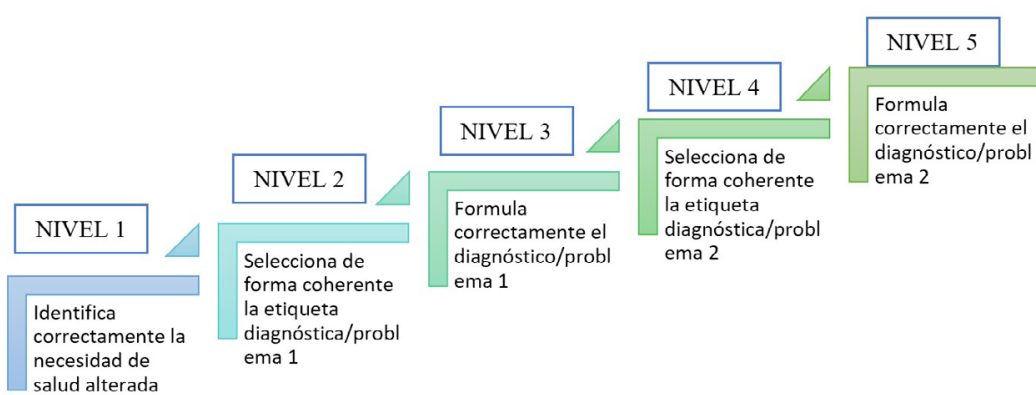

Figura 4. Escalera de aprendizaje de la fase diagnóstico

Pregunta: ¿Cómo voy a abordarlo? ¿Qué resultados espero? ¿Qué puedo hacer?

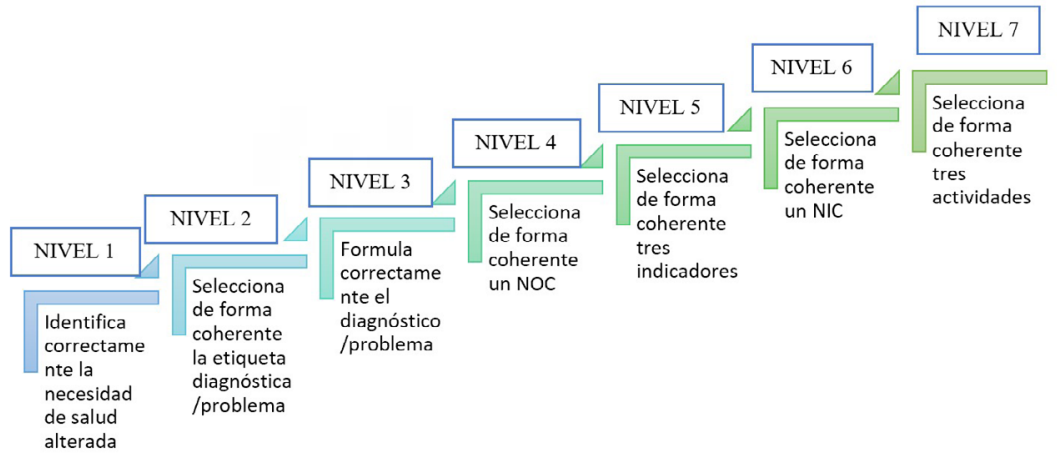

Figura 5. Escalera de aprendizaje de la fase planificación

\section{Aplicación del ciclo de mejora docente}

\section{Relato de las sesiones}

Las sesiones se desarrollaron del modo esperado en líneas generales. Se distribuye al alumnado el caso práctico con unas preguntas para resolver y un material de apoyo. Se les recomienda consultar material adicional en internet. El alumnado trabaja en equipo el caso clínico y se corrige en común obteniendo una retroalimentación que 
genera el aprendizaje. A pesar de las explicaciones previas, la primera sesión generó desconcierto y algo de rechazo por parte del alumnado, por tratarse de una metodología diferente a la que no estaban acostumbrados. Con el transcurso de las sesiones, se adaptaron al cambio de enfoque hacia un aprendizaje autónomo y fueron asumiendo un papel más protagonista.

En relación con la actuación docente, este CMD ha supuesto un cambio de mentalidad que ha requerido frenar el impulso corrector por parte de la docente. De forma espontánea la intuición de indicarles lo que debían hacer, pero se evitó para no influir en el proceso de descubrimiento propio por parte del alumnado.

El desarrollo de las sesiones hizo necesario introducir una modificación con respecto al diseño para adaptarse a la reacción del alumnado. En la primera sesión se utilizó la plataforma Kahoot para corregir el caso clínico, como estaba previsto. El alumnado contaba con experiencias anteriores con esta plataforma, en otras asignaturas la han empleado para repaso de los temas. La experiencia del alumnado consistía en utilizarla como un concurso competitivo, sin retroalimentación para corregir errores. Este enfoque estaba tan arraigado que se optó por otros métodos de corrección de los casos clínicos.

\section{Evaluación del aprendizaje de los estudiantes}

La evolución del aprendizaje del alumnado es apreciable al comparar los conocimientos iniciales y finales del alumnado, a través de las escaleras de aprendizaje. Los casos clínicos fueron realizados por una medica de 22,3 equipos de trabajo configurado por 2-3 personas. La demostración de conocimientos es progresiva a medida que se avanza en los casos, como se aprecia en la Tabla 3, de modo que los conocimientos finales son ampliamente superiores a los iniciales. 
Tabla 3. Porcentaje de equipos de trabajo que han demostrado los conocimientos de cada nivel

\begin{tabular}{|c|c|c|c|c|c|c|}
\hline & Caso 1 & Caso 2 & Caso 3 & Caso 4 & Caso 5 & Caso 6 \\
\hline $\begin{array}{c}\text { Número de } \\
\text { equipos de } \\
\text { trabajo }\end{array}$ & 20 & 24 & 24 & 23 & 21 & 22 \\
\hline Nivel 1 & $15,0 \%$ & $33,3 \%$ & $45,8 \%$ & $87,0 \%$ & $90,5 \%$ & $95,5 \%$ \\
\hline Nivel 2 & $55,0 \%$ & $87,5 \%$ & $83,3 \%$ & $91,3 \%$ & $71,4 \%$ & $90,9 \%$ \\
\hline Nivel 3 & $35,0 \%$ & $41,7 \%$ & $25,0 \%$ & $87,0 \%$ & $71,4 \%$ & $90,9 \%$ \\
\hline Nivel 4 & $90,0 \%$ & $58,3 \%$ & $12,5 \%$ & $39,1 \%$ & $90,5 \%$ & $81,8 \%$ \\
\hline Nivel 5 & $5,0 \%$ & $25,0 \%$ & $16,7 \%$ & $21,7 \%$ & $14,3 \%$ & $54,5 \%$ \\
\hline Nivel 6 & $*$ & $*$ & $*$ & $100,0 \%$ & $85,7 \%$ & $86,4 \%$ \\
\hline Nivel 7 & $*$ & $*$ & $*$ & $30,4 \%$ & $4,8 \%$ & $54,5 \%$ \\
\hline
\end{tabular}

* La escalera de aprendizaje de estos casos contaba solamente con 5 niveles.

\section{Evaluación del ciclo de mejora docente}

Para la evaluación del propio CMD se emplean los siguientes métodos:

- Observación directa y cuaderno de campo: durante las sesiones de casos clínicos, la profesora observa y valora su desarrollo tomando notas en el cuaderno de campo. Estas notas servirán como orientaciones de mejora intermedias para la sesión siguiente adaptando cada sesión a las dificultades o particularidades de cada grupo.

- Cuestionario de satisfacción: al finalizar la última sesión, se distribuirá un cuestionario de satisfacción a los estudiantes para valorar los puntos fuertes y débiles del diseño. También se les solicitará propuestas de mejora. Las respuestas de este cuestionario nos orientan sobre la satisfacción, grado de acogida y motivación del alumnado

Jornadas de Formación e Innovación Docente del Profesorado | № 1 (2018) Esta obra se distribuye con la licencia Creative Commons 
- Preguntas de reflexión: para evaluar el modelo de aprendizaje del alumnado y fomentar la reflexión sobre su propio aprendizaje, al finalizar se solicita al alumnado que respondan a través del campus virtual las siguientes preguntas: ¿qué he aprendido? y ¿qué necesidad de aprendizaje me genera?. Con estas preguntas se pretende fomentar el pensamiento crítico y concienciar al alumnado sobre su aprendizaje.

El cuestionario de satisfacción lo rellenaron voluntariamente y de forma anónima los asistentes a la última sesión, es decir, un grupo formado por 54 participantes de los cuales el $72 \%$ fueron mujeres y el $28 \%$ hombre, de una edad media de 20,4 años. En relación con la satisfacción del alumnado, el mayor de grado de acuerdo se obtuvo al afirmar que el ABP potencia el trabajo en equipo, facilita el pensamiento crítico y logra una mejor comprensión de la materia, como se puede observar en la Figura 6.

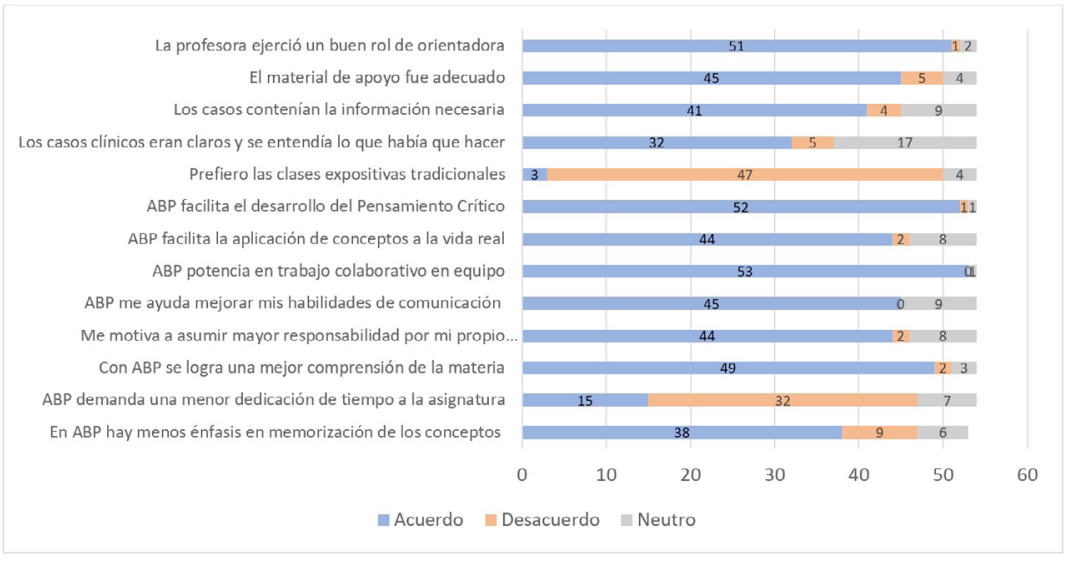

Figura 6. Satisfacción del alumnado.

En relación con las preguntas de reflexión sobre el propio aprendizaje, las respuestas a la pregunta ¿Qué he aprendido? se han sintetizado y agrupado de acuerdo con el mapa de contenidos planteado previamente, en conceptuales, procedimentales y actitudinales. Como se observa 

cipalmente actitudes con esta modalidad de enseñanza.

\section{Conceptuales}

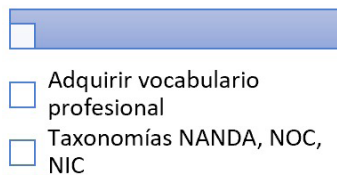

NIC

\section{Procedimentales}

$\square$ Abordar problemas de los
$\square$ pacientes de forma
efectiva
$\square$ Elaborar planes de cuidado
$\square$ Emplear recursos
electrónicos
$\square$ Emplear taxonomía
enfermera
$\square \begin{aligned} & \text { Aplicar la teoría a la } \\ & \text { práctica }\end{aligned}$
$\square \begin{aligned} & \text { Priorizar problemas de los } \\ & \text { pacientes }\end{aligned}$
$\square \begin{aligned} & \text { Interpretar datos del } \\ & \text { paciente }\end{aligned}$

Actitudinales

$\square$ Trabajar con mis
compañeros
$\square$ Desarrollar pensamiento
crítico
Confianza en mí misma y
seguridad
$\square$ Motivación, ilusión, ganas
de aprender
$\square$ Escuchar las opiniones de
los demás
$\square$ Razonar los problemas
$\square$ Tomar decisiones
$\square \begin{aligned} & \text { Considerar más de una } \\ & \text { opción antes de decidir }\end{aligned}$
$\square$ Aprender del error
$\square \begin{aligned} & \text { Llegar a un consenso con } \\ & \text { mis compañeros }\end{aligned}$
$\square \begin{aligned} & \text { Considerar soluciones } \\ & \text { sencillas }\end{aligned}$
$\begin{aligned} & \text { Resolver problemas con } \\ & \text { calma }\end{aligned}$
$\square \begin{aligned} & \text { Aportar argumentos a mis } \\ & \text { opiniones }\end{aligned}$
$\square$ Autocrítica

Figura 7. Reflexiones del alumnado sobre su propio aprendizaje

En relación con la pregunta ¿qué necesidad de aprendizaje me genera?, el alumnado identificó las siguientes carencias propias, como áreas de mejora del aprendizaje:

- Ganas de aprender más

- Falta de confianza en mí misma

- Practicar más casos clínicos

- Mejorar la lectura comprensiva

- Aprender a identificar el problema del paciente

- Destreza informática

- Dar más valor a mis opiniones

- Manejo y aplicación de las taxonomías NANDA, NOC, NIC

- Empatía y actitud asertiva 
- Toma de decisiones

- Pensamiento crítico

- Resolución de problemas

- Comunicación, transmitir información de forma eficiente

- Argumentar mis opiniones

- Desenvolverme en situaciones clínicas

- Aprender sobre el rol de enfermería

\section{Conclusiones}

El sistema de aprendizaje basado en casos clínicos ha mostrado ser efectivo especialmente para los contenidos actitudinales. Tomar como punto de partida una situación desconocida genera la búsqueda de posibles soluciones, compartir opiniones con los compañeros, argumentarlas y elegir una opción de forma crítica y consensuada. El empleo del error como herramienta docente fomenta la motivación del alumnado y sirve de estímulo para el aprendizaje.

En futuros ciclos de mejora más amplios que abarquen a toda la asignatura se recomienda incorporar la metodología de casos clínicos, aunque mejorando la forma de evaluación y puesta en común de los casos. El cuaderno de campo ha resultado especialmente útil para identificar la necesidad de modificaciones y adaptarse a la realidad del grupo y a su reacción ante la metodología.

Jornadas de Formación e Innovación Docente del Profesorado | № 1 (2018) Esta obra se distribuye con la licencia Creative Commons 


\section{Referencias}

Gonzánez, C., Carbonero, M., Lara, F., \& Martín, P. (2014). Aprendizaje basado en problemas y satisfacción de los estudiantes de Enfermería. Enferm Glob, 13(35), 97-103. Retrieved from http://search.ebscohost.com/login.aspx? direct=true \& $\mathrm{db}=1$ th \&AN $=96903106 \&$ lang $=e s \&$ site $=e-$ host-live

Latasa, I., Lozano, P., \& Ocerinjauregi, N. (2012). Aprendizaje Basado en Problemas en Currículos Tradicionales: Beneficios e Inconvenientes. Formación Universitaria, 5(5), 15-26. https://doi.org/10.4067/ S0718-50062012000500003

Martín, G., Martínez, R., Martín, M., \& Nieto, M. (2017). Acercamiento a las Teorías del Aprendizaje en la Educación Superior. Uniandes, 4(1), 48-60. Retrieved from http://186.46.158.26/ojs/index.php/EPISTEME/article/ view/346

Prieto Martin, A., Barbarroja Escudero, J., Reyes Martin, E., Monserrat Sanz, J., Marti, D., N, D., ... Ivarez-Mon Soto, M. (2006). Un nuevo modelo de aprendizaje basado en problemas, el ABP 4x4, es eficaz para desarrollar competencias profesionales valiosas en asignaturas con más de 100 alumnos. Aula Abierta, 87(87), 171-194. https://doi.org/10.1007/s11434-011-4778-3

Sánchez, I. R., \& Ramis, F. J. (2004). Aprendizaje significativo basado en problemas. Horizontes Educacionales, (9), 101-111. https://doi.org/10.5944/ried.16.1.2060 